PROCEEDINGS OF THE

AMERICAN MATHEMATICAL SOCIETY

Volume 126, Number 10, October 1998, Pages 3027-3036

S 0002-9939(98)04415-3

\title{
SUMMABILITY OF FOURIER ORTHOGONAL SERIES FOR JACOBI WEIGHT FUNCTIONS ON THE SIMPLEX IN $\mathbb{R}^{d}$
}

\author{
YUAN XU
}

(Communicated by Palle E. T. Jorgensen)

\begin{abstract}
We study the Fourier expansion of a function in orthogonal polynomial series with respect to the weight functions

$$
x_{1}^{\alpha_{1}-1 / 2} \cdots x_{d}^{\alpha_{d}-1 / 2}\left(1-|\mathbf{x}|_{1}\right)^{\alpha_{d+1}-1 / 2}
$$

on the standard simplex $\Sigma^{d}$ in $\mathbb{R}^{d}$. It is proved that such an expansion is uniformly $(C, \delta)$ summable on the simplex for any continuous function if and only if $\delta>|\alpha|_{1}+(d-1) / 2$. Moreover, it is shown that $\left(C,|\alpha|_{1}+(d+1) / 2\right)$ means define a positive linear polynomial identity, and the index is sharp in the sense that $(C, \delta)$ means are not positive for $0<\delta<|\alpha|_{1}+(d+1) / 2$.
\end{abstract}

\section{INTRODUCTION AND MAIN RESULTS}

The purpose of this paper is to study the Cesàro summability of the Fourier orthogonal series with respect to the weight function

$$
W_{\alpha}(\mathbf{x})=w_{\alpha} x_{1}^{\alpha_{1}-1 / 2} \cdots x_{d}^{\alpha_{d}-1 / 2}\left(1-|\mathbf{x}|_{1}\right)^{\alpha_{d+1}-1 / 2}, \quad \alpha_{i} \geq 0
$$

on the standard simplex $\Sigma^{d}:=\left\{\mathbf{x} \in \mathbb{R}^{d}: x_{1} \geq 0, \ldots, x_{d} \geq 0,1-|\mathbf{x}|_{1} \geq 0\right\}$ in $\mathbb{R}^{d}$, where $w_{\alpha}$ is a normalization constant such that $\int_{\Sigma^{d}} W_{\alpha} d \mathbf{x}=1$. For $d=1$ this is the summability with respect to the Jacobi weight function on $[0,1]$. In particular, in the case of $d=1, \alpha_{1}=\alpha_{2}=0$, it is the same as the summability of the Fourier cosine series, for which the classical result states that the Cesàro $(C, \delta)$ means are uniformly summable for any continuous function if and only if $\delta>0$; moreover, the $(C, 1)$ means of the Fourier cosine series define a positive linear operator whose kernel is the well-known Fejér kernel. The purpose of this paper is to prove analogues of these results for $W_{\alpha}$ on $\Sigma^{d}, d>1$.

Let $\Pi^{d}$ be the space of polynomials in $d$ variables and $\Pi_{n}^{d}$ be the subspace of polynomials of degree at most $n$. We also denote by $\mathcal{P}_{n}^{d}$ the space of homogeneous polynomials of degree $n$ on $\mathbb{R}^{d}$, and we let $r_{n}^{d}=\operatorname{dim} \mathcal{P}_{n}^{d}$. It is known that $r_{n}^{d}=\left(\begin{array}{c}n+d-1 \\ n\end{array}\right)$. We denote by $\left\{P_{k}^{n}\right\}, 1 \leq k \leq r_{n}^{d}$ and $0 \leq n<\infty$, one family of orthonormal polynomials with respect to $W_{\alpha}$ that forms a basis of $\Pi_{n}^{d}$, where the superscript $n$ means that $P_{k}^{n} \in \Pi_{n}^{d}$. Let $f$ be integrable with respect to $W_{\alpha}$.

Received by the editors March 14, 1997.

1991 Mathematics Subject Classification. Primary 33C50, 42C05, 41A63.

Key words and phrases. Orthogonal polynomials in several variables, on simplex, Cesàro summability, positive kernel.

Supported by the National Science Foundation under Grant DMS-9500532.

(C)1998 American Mathematical Society 
The Fourier orthogonal expansion of $f$ with respect to a sequence of orthonormal polynomials $\left\{P_{k}^{n}\right\}$ is given by

$$
f \sim \sum_{n=0}^{\infty} \sum_{k=1}^{r_{n}^{d}} a_{k}^{n}(f) P_{k}^{n}(\mathbf{x}), \quad \text { where } \quad a_{k}^{n}(f)=\int P_{k}^{n}(\mathbf{x}) f(\mathbf{x}) W_{\alpha}(\mathbf{x}) d \mathbf{x}
$$

Let $\mathcal{V}_{n}^{d}$ denote the subspace spanned by the polynomials $P_{k}^{n}, 1 \leq k \leq r_{n}^{d}$. Although for $d>1$ the orthonormal basis of $\Pi^{d}$ is not unique, the Fourier orthogonal expansion is unique if we consider it as an expansion in terms of the subspaces $\mathcal{V}_{0}^{d}, \mathcal{V}_{1}^{d}, \ldots$ (cf. [9]). Let us denote the reproducing kernel of $\mathcal{V}_{n}^{d}$ by $\mathbf{P}_{n}\left(W_{\alpha}\right)$ and the $n$-th reproducing kernel of $\Pi^{d}$ by $\mathbf{K}_{n}\left(W_{\alpha}\right)$. It follows that

$$
\mathbf{P}_{n}\left(W_{\alpha} ; \mathbf{x}, \mathbf{y}\right)=\mathbb{P}_{n}^{T}(\mathbf{x}) \mathbb{P}_{n}(\mathbf{y}), \quad \text { and } \quad \mathbf{K}_{n}\left(W_{\alpha} ; \mathbf{x}, \mathbf{y}\right)=\sum_{k=0}^{n} \mathbf{P}_{k}\left(W_{\alpha} ; \mathbf{x}, \mathbf{y}\right)
$$

where we use the vector notation $\mathbb{P}_{n}=\left(P_{1}^{n}, \ldots, P_{r_{n}^{d}}^{n}\right)^{T}$. Since any two orthonormal bases of $\mathcal{V}_{n}^{d}$ differ by an orthogonal matrix, both $\mathbf{P}_{n}\left(W_{\alpha} ; \mathbf{x}, \mathbf{y}\right)$ and $\mathbf{K}_{n}\left(W_{\alpha} ; \mathbf{x}, \mathbf{y}\right)$ are independent of the choice of orthonormal basis.

We denote by $S_{n}\left(W_{\alpha} ; f\right)$ the $n$-th partial sum of the Fourier orthogonal series of $f$ with respect to $W_{\alpha}$ on $\Sigma^{d}$. By (1.2) and (1.3), we have

$$
S_{n}\left(W_{\alpha} ; f, \mathbf{x}\right)=\sum_{k=0}^{n} \sum_{j} a_{j}^{k}(f) P_{j}^{k}(\mathbf{x})=\int_{\Sigma^{d}} f(\mathbf{y}) \mathbf{K}_{n}\left(W_{\alpha} ; \mathbf{x}, \mathbf{y}\right) W_{\alpha}(\mathbf{y}) d \mathbf{y} .
$$

In this paper we will derive compact formulae for $\mathbf{P}_{n}\left(W_{\alpha}\right)$ and $\mathbf{K}_{n}\left(W_{\alpha}\right)$ and use them to study the $(C, \delta)$ means of $S_{n}\left(W_{\alpha} ; f\right)$. The main results are as follows.

Theorem 1.1. Let $f$ be continuous on the simplex $\Sigma^{d}$. The Cesàro $(C, \delta)$ means of the expansion of $f$ in the Fourier orthogonal series with respect to $W_{\alpha}$ converge uniformly to $f$ on $\Sigma^{d}$ if, and only if, $\delta>|\alpha|_{1}+(d-1) / 2$.

Theorem 1.2. The Cesàro $\left(C,|\alpha|_{1}+(d+1) / 2\right)$ means of the expansion of a function in the Fourier orthogonal series with respect to $W_{\alpha}$ define a positive linear polynomial approximation identity on $C\left(\Sigma^{d}\right)$; the order of summability is best possible in the sense that the $(C, \delta)$ means are not positive for $0<\delta<|\alpha|_{1}+(d+1) / 2$.

We note that for $d=1$ and $\alpha=0$, these two theorems agree with the well-known results for Fourier cosine series. There are other extensions of the Fejér kernel to higher dimensions; see [2] for an example. Let us recall the definition of Cesàro's summability (cf. [13, Chapt. 3]). For $\delta>0$, the Cesàro $(C, \delta)$ means, $s_{n}^{\delta}$, of a sequence $\left\{s_{n}\right\}$ are defined by

$$
s_{n}^{\delta}=\frac{1}{\left(\begin{array}{c}
n+\delta \\
n
\end{array}\right)} \sum_{k=0}^{n}\left(\begin{array}{c}
n-k+\delta-1 \\
n-k
\end{array}\right) s_{k}=\frac{1}{\left(\begin{array}{c}
n+\delta \\
n
\end{array}\right)} \sum_{k=0}^{n}\left(\begin{array}{c}
n-k+\delta \\
n-k
\end{array}\right) c_{k},
$$

where the second equality holds if $s_{n}$ is the $n$-th partial sum of the series $\sum_{k=0}^{\infty} c_{k}$. We say that $\left\{s_{n}\right\}$ is Cesàro $(C, \delta)$ summable to $s$ if $s_{n}^{\delta}$ converges to $s$ as $n \rightarrow \infty$.

The proofs of the theorems use several recent results. The key ingredient is a connection between orthogonal polynomials on $\Sigma^{d}$ and homogeneous orthogonal polynomials on the unit sphere $S^{d}$ established in [12]. Under this connection, orthogonal polynomials with respect to the weight function $W_{\alpha}$ on $\Sigma^{d}$ correspond 
to orthogonal polynomials invariant under the group $\left(\mathbb{Z}_{2}\right)^{d+1}$ with respect to the weight function $h_{\alpha}^{2} d \omega$ on $S^{d}$, where

$$
h_{\alpha}(\mathbf{y})=H_{\alpha}\left|\mathbf{y}_{1}\right|^{\alpha_{1}} \ldots\left|\mathbf{y}_{d+1}\right|^{\alpha_{d+1}}, \quad \mathbf{y} \in \mathbb{R}^{d+1},
$$

and $H_{\alpha}$ is a constant so that $\int_{S^{d}} h_{\alpha}^{2} d \omega=1$; the $h_{\alpha}$ is invariant under the same group. The orthogonal structure on $S^{d}$ with respect to $h_{\alpha}^{2} d \omega$ has been studied in detail in [10] as a special case of Dunkl's theory of $h$-harmonics for measures invariant under finite reflection groups; the $h$-harmonics are homogeneous orthogonal polynomials with respect to $h^{2} d \omega$. The connection and the results in [10] makes it possible to derive a compact formula for $\mathbf{P}_{n}\left(W_{\alpha}\right)$, which is written as an integral with respect to a Gegenbauer polynomial. The integral can also be written in terms of the intertwining operator in Dunkl's theory. The proof of Theorem 1.1 uses a general theorem in [11] about the integral of the intertwining operator. The positivity in Theorem 1.2 uses an Askey-Gasper inequality ([1], [6]).

In the following section we discuss orthogonal polynomials with respect to $W_{\alpha}$ and derive the compact formulae for $\mathbf{P}_{n}\left(W_{\alpha}\right)$. The proof of the theorems is in Section 3 .

\section{Orthogonal StRuCture AND COMPACT FORMUlaE FOR REPRODUCING KERNEL}

We start with the connection between orthogonal polynomials on $\Sigma^{d}$ and those on $S^{d}$, which serves as the foundation of our development. For $\mathbf{x}, \mathbf{y} \in \mathbb{R}^{d}$ we denote their inner product by $\langle\mathbf{x}, \mathbf{y}\rangle=x_{1} y_{1}+\ldots+x_{d} y_{d}$; we denote by $|\mathbf{x}|=\sqrt{\langle\mathbf{x}, \mathbf{x}\rangle}$ the usual Euclidean norm. Let $B^{d}=\left\{\mathbf{x} \in \mathbb{R}^{d}:|\mathbf{x}| \leq 1\right\}$ be the unit ball of $\mathbb{R}^{d}$ and $S^{d}=\left\{\mathbf{y} \in \mathbb{R}^{d+1}:|\mathbf{y}|=1\right\}$ be the unit sphere on $\mathbb{R}^{d+1}$. We also denote the $\ell^{1}$ norm of $\mathbf{x} \in \mathbb{R}^{d}$ by $|\mathbf{x}|_{1}=\left|x_{1}\right|+\cdots+\left|x_{d}\right|$. The connection between $\Sigma^{d}$ and $S^{d}$ is based on the following integral formula:

$$
\int_{S^{d}} f\left(y_{1}^{2}, \ldots, y_{d+1}^{2}\right) d \omega=2 \int_{\Sigma^{d}} f\left(\mathbf{x}, 1-|\mathbf{x}|_{1}\right)\left(x_{1} \cdots x_{d}\left(1-|\mathbf{x}|_{1}\right)\right)^{-1 / 2} d \mathbf{x}
$$

which will be useful in our later development as well. This formula can be easily verified by changing variables (cf. [3], [12]). It implies, in particular, that

$$
\int_{S^{d}} f\left(y_{1}^{2}, \ldots, y_{d+1}^{2}\right) h_{\alpha}^{2}(\mathbf{y}) d \omega=\int_{\Sigma^{d}} f\left(\mathbf{x}, 1-|\mathbf{x}|_{1}\right) W_{\alpha}(\mathbf{x}) d \mathbf{x}
$$

by (1.1) and (1.6). We use this formula to connect orthogonal polynomials for $W_{\alpha}$ on $\Sigma^{d}$ to $h$-harmonics for $h_{\alpha}^{2}$ on $S^{d}$. Let us first recall the basics of Dunkl's theory; we restrict the statement to the special case under consideration.

The key ingredient of the theory is a family of commuting first-order differentialdifference operators $\mathcal{D}_{i}$ (Dunkl's operators), which act very much like the partial derivatives $\partial_{i}([4])$. In the present much restricted case, the operators take the form

$$
\mathcal{D}_{j} f(\mathbf{x})=\partial_{j} f(\mathbf{x})+\alpha_{j} \frac{f(\mathbf{x})-f\left(\mathbf{x} \sigma_{j}\right)}{x_{j}}, \quad 1 \leq j \leq d,
$$

where $\mathbf{x} \sigma_{j}=\left(x_{1}, \ldots, x_{j-1},-x_{j}, x_{j+1}, \ldots, x_{d+1}\right)$. The $h$-Laplacian, which plays the role similar to that of the usual Laplacian, is defined by $\Delta_{h}=\mathcal{D}_{1}^{2}+\ldots+\mathcal{D}_{d}^{2}$. It is proved that if $P \in \mathcal{P}_{n}^{d}$, then $\int_{S^{d-1}} P Q h_{\alpha}^{2} d \omega=0$ for any $Q$ of degree less than $n$ if and only if $\Delta_{h} P=0$. The space $\mathcal{H}_{n}\left(h^{2}\right):=\mathcal{P}_{n}^{d} \cap \operatorname{ker} \Delta_{h}$ is called the space of $h$-harmonic polynomials of degree $n$. The weight function $h_{\alpha}$ in (1.6) is invariant 
under the group $G=\left(\mathbb{Z}_{2}\right)^{d+1}$. The element of $G$ takes the form $\varepsilon=\left(\varepsilon_{1}, \ldots, \varepsilon_{d+1}\right)$, where $\varepsilon_{i}= \pm 1$. For a function $f$ defined on $\mathbb{R}^{d+1}$, the action of $\varepsilon \in G$ on $f$ is defined by $R(\varepsilon) f(\mathbf{x})=f(\mathbf{x} \varepsilon)=f\left(x_{1} \varepsilon_{1}, \ldots, x_{d+1} \varepsilon_{d+1}\right)$. If $R(\varepsilon) f=f$ for all $\varepsilon \in G$, we say that $f$ is invariant under the group $G$. We denote by $\mathcal{H}_{2 n}^{G}$ the subspace of $h$-harmonics of degree $2 n$ that are invariant under $G$.

We will also need the following notation: For $\mathbf{x} \in \mathbb{R}^{d+1}$, we write

$$
\mathbf{x}=\left(x_{1}, \ldots, x_{d}, x_{d+1}\right)=r \mathbf{u}^{\prime}=r\left(\mathbf{u}, u_{d+1}\right), \quad \mathbf{u}^{\prime} \in S^{d}, \quad \mathbf{u} \in B^{d},
$$

where $r=|\mathbf{x}|$. We note that if $\mathbf{u} \in B^{d}$, then $\left(u_{1}^{2}, \ldots, u_{d}^{2}\right) \in \Sigma^{d}$; moreover, $u_{d+1}=$ $\sqrt{1-|\mathbf{u}|^{2}}$. The following lemma is proved using the formula (2.2) (cf. [12]).

Lemma 2.1. Let $\left\{P_{k}^{n}\right\}$ be a sequence of orthonormal polynomials with respect to $W_{\alpha}$ on $\Sigma^{d}$. Let $S_{k}^{2 n}$ be polynomials of $d+1$ variables defined, according to (2.3), by

$$
S_{k}^{2 n}(\mathbf{x})=|\mathbf{x}|^{2 n} P_{k}^{n}\left(u_{1}^{2}, \ldots, u_{d}^{2}\right), \quad 1 \leq k \leq r_{n}^{d} .
$$

Then $\left\{S_{k}^{2 n}\right\}$ forms an orthonormal basis for $\mathcal{H}_{2 n}^{G}$ with respect to $h_{\alpha}$ in (1.6).

In particular, $S_{k}^{2 n}$ are homogeneous of degree $2 n$. Moreover, if an $h$-harmonic polynomial of degree $2 n$ takes the form (2.4), then the polynomial $P_{k}^{n}(\mathbf{x})$ is an orthonormal polynomial with respect to $W_{\alpha}$ on $\Sigma^{d}$. In an effort to understand Dunkl's theory of $h$-harmonics associated with general reflection groups, we studied as an example the $h$-harmonics associated to $h_{\alpha}$ in detail in [10]. The results include closed formulae for an orthonormal basis, for the reproducing kernel, and for the intertwining operator.

The intertwining operator $V$ intertwines the algebra generated by $\mathcal{D}_{i}$ and the algebra of partial differential operators. It is uniquely determined by

$$
V \mathcal{P}_{n} \subset \mathcal{P}_{n}, \quad V 1=1, \quad \mathcal{D}_{i} V=V \partial_{i}, \quad 1 \leq i \leq d .
$$

Although the closed formula for $V$ for a general reflection group is not known, in the case $\left(\mathbb{Z}_{2}\right)^{d+1}$ it is shown in [10] (cf. [5] for $d=2$ ) that

$$
V f(\mathbf{y})=\int_{[-1,1]^{d+1}} f\left(y_{1} t_{1}, \ldots, y_{d+1} t_{d+1}\right) \prod_{i=1}^{d+1}\left(1+t_{i}\right) \prod_{i=1}^{d+1} c_{\alpha_{i}}\left(1-t_{i}^{2}\right)^{\alpha_{i}-1} d \mathbf{t},
$$

where $c_{\mu}=1 / \int_{-1}^{1}\left(1-t^{2}\right)^{\mu-1} d t$, which can be easily verified by the definition of $V$. The reproducing kernel $P_{n}^{h}$ of the $h$-harmonics $\mathcal{H}_{n}\left(h^{2}\right)$ is given by ([5], [10])

$$
\begin{aligned}
P_{n}^{h}(\mathbf{x}, \mathbf{y})= & \frac{n+|\alpha|_{1}+\frac{d-1}{2}}{|\alpha|_{1}+\frac{d-1}{2}}\left[V C_{n}^{\left(|\alpha|_{1}+(d-1) / 2\right)}(\langle\cdot, \mathbf{y}\rangle)\right](\mathbf{x}) \\
= & \frac{n+|\alpha|_{1}+\frac{d-1}{2}}{|\alpha|_{1}+\frac{d-1}{2}} \\
& \times \int_{[-1,1]^{d+1}} C_{n}^{\left(|\alpha|_{1}+(d-1) / 2\right)}\left(x_{1} y_{1} t_{1}+\ldots+x_{d+1} y_{d+1} t_{d+1}\right) \prod_{i=1}^{d+1}\left(1+t_{i}\right) \\
& \times \prod_{i=1}^{d+1} c_{\alpha_{i}}\left(1-t_{i}^{2}\right)^{\alpha_{i}-1} d \mathbf{t},
\end{aligned}
$$


where $C_{n}^{(\lambda)}$ denotes the Gegenbauer polynomial of degree $n$ (cf. [8], where the notation $P_{n}^{(\lambda)}$ is used). If $\left\{Y_{k}^{n}\right\}$ forms an orthonormal basis of $\mathcal{H}_{n}\left(h^{2}\right)$, then

$$
P_{n}^{h}(\mathbf{x}, \mathbf{y})=\sum_{k=1}^{N_{n}} Y_{k}^{n}(\mathbf{x}) Y_{k}^{n}(\mathbf{y}), \quad N_{n}=\operatorname{dim} \mathcal{H}_{n}\left(h^{2}\right) .
$$

We can also use (2.4) and the results in [10] to write down closed formulae for an orthonormal basis, which are not needed, however, for deriving the compact formula for the reproducing kernel with respect to $W_{\Sigma}$. We have

Theorem 2.2. The $n$-th reproducing kernel $\mathbf{P}_{n}\left(W_{\alpha} ; \cdot, \cdot\right)$ of $\mathcal{V}_{n}^{d}$ is given by

$$
\begin{aligned}
\mathbf{P}_{n}\left(W_{\alpha} ; \mathbf{x}, \mathbf{y}\right)=\frac{2 n+|\alpha|_{1}+\frac{d-1}{2}}{2^{d+1}\left(|\alpha|_{1}+\frac{d-1}{2}\right)} & \\
\times \int_{[-1,1]^{d+1}} C_{2 n}^{\left(|\alpha|_{1}+(d-1) / 2\right)}\left(\sqrt{x_{1} y_{1}} t_{1}\right. & \left.+\ldots+\sqrt{x_{d+1} y_{d+1}} t_{d+1}\right) \\
\times & \times \prod_{i=1}^{d+1} c_{\alpha_{i}}\left(1-t_{i}^{2}\right)^{\alpha_{i}-1} d \mathbf{t},
\end{aligned}
$$

where $\mathbf{x}, \mathbf{y} \in \Sigma^{d}, x_{d+1}=1-|\mathbf{x}|_{1}$ and $y_{d+1}=1-|\mathbf{y}|_{1}$.

Proof. First we claim that the reproducing kernel of $\mathcal{H}_{2 n}^{G}$ is given by

$$
Z_{2 n}^{G}(\mathbf{x}, \mathbf{y})=2^{-d-1} \sum_{\varepsilon \in G}|\mathbf{x}|^{2 n}|\mathbf{y}|^{2 n} P_{2 n}^{h}(\mathbf{x}, \mathbf{y} \varepsilon), \quad \mathbf{x}, \mathbf{y} \in \mathbb{R}^{d} .
$$

Indeed, if $P$ is an invariant harmonic polynomial of degree $2 n$, then it is easy to see that

$$
\int_{S^{d}} Z_{2 n}^{G}(\mathbf{x}, \mathbf{y}) P(\mathbf{y}) h_{\alpha}^{2}(\mathbf{y}) d \omega=\int_{S^{d}} P_{2 n}^{h}(\mathbf{x}, \mathbf{y}) P(\mathbf{y}) h_{\alpha}^{2}(\mathbf{y}) d \omega=P(\mathbf{x}) ;
$$

if $P$ is not invariant under $G$, then the integral is zero since $P$ must be an odd function with respect to at least one of its variables. We can also write down $Z_{2 n}^{G}$ according to an orthonormal basis of $\mathcal{H}_{2 n}^{G}$. Recall the notation (2.3); we write $\mathbf{u}^{\prime}=\left(\mathbf{u}, u_{d+1}\right)$ and $\mathbf{v}^{\prime}=\left(\mathbf{v}, v_{d+1}\right)$ with $\left|\mathbf{u}^{\prime}\right|=\left|\mathbf{v}^{\prime}\right|=1$. Then, since an orthonormal basis of $\mathcal{V}_{n}^{d}$ with respect to $W_{\alpha}$ defines an orthonormal basis for $\mathcal{H}_{2 n}^{G}$ via $(2.4)$, it follows that

$\mathbf{P}_{n}\left(W_{\alpha} ;\{\mathbf{u}\}^{2},\{\mathbf{v}\}^{2}\right)=\sum_{k} P_{k}^{n}\left(\{\mathbf{u}\}^{2}\right) P_{k}^{n}\left(\{\mathbf{v}\}^{2}\right)=\sum_{k} S_{k}^{2 n}\left(\mathbf{u}^{\prime}\right) S_{k}^{2 n}\left(\mathbf{v}^{\prime}\right)=Z_{2 n}^{G}\left(\mathbf{u}^{\prime}, \mathbf{v}^{\prime}\right)$,

where we define $\{\mathbf{u}\}^{2}=\left(u_{1}^{2}, \ldots, u_{d}^{2}\right)$. We note that $\left|\mathbf{u}^{\prime}\right|=1$ implies that $\{\mathbf{u}\}^{2} \in \Sigma^{d}$. Upon taking square roots, we see that the correspondence between $\{\mathbf{u}\}^{2} \in \Sigma^{d}$ and $\mathbf{u}^{\prime}=\left(\mathbf{u}, u_{d+1}\right) \in S^{d}$ in the above formula becomes the one between $\mathbf{u} \in \Sigma^{d}$ and $\left\{\mathbf{u}^{\prime}\right\}^{1 / 2}=\left(u_{1}^{1 / 2}, \ldots, u_{d+1}^{1 / 2}\right)=\left(\{\mathbf{u}\}^{1 / 2}, u_{d+1}^{1 / 2}\right) \in S^{d}$, where $u_{d+1}=1-\left|\{\mathbf{u}\}^{1 / 2}\right|^{2}=$ $1-|\mathbf{u}|_{1}$. Hence, the above formula, together with (2.6) and the formula for $P_{n}^{h}$, allows us to write for $\mathbf{u}, \mathbf{v} \in \Sigma^{d}$,

$$
\mathbf{P}_{n}\left(W_{\alpha} ; \mathbf{u}, \mathbf{v}\right)=\frac{2 n+|\alpha|_{1}+\frac{d-1}{2}}{2^{d+1}\left(|\alpha|_{1}+\frac{d-1}{2}\right)} \sum_{\varepsilon \in G} V\left[C_{2 n}^{\left(|\alpha|_{1}+(d-1) / 2\right)}\left(\left\langle\cdot,\left\{\mathbf{u}^{\prime}\right\}^{1 / 2} \varepsilon\right\rangle\right)\right]\left(\left\{\mathbf{v}^{\prime}\right\}^{1 / 2}\right) .
$$


Using the formula for $P_{n}^{h}$, we can write $\mathbf{P}_{n}\left(W_{\alpha} ; \mathbf{u}, \mathbf{v}\right)$ as an integral, which gives the desired formula for $\mathbf{P}_{n}\left(W_{\alpha}\right)$ upon changing variables $t_{i} \mapsto-t_{i}$ whenever $\epsilon_{i}=$ -1 .

Theorem 2.3. Usig the notation of Theorem 2.2, the kernel $\mathbf{K}_{n}\left(W_{\alpha}\right)$ is given by

$$
\begin{aligned}
\mathbf{K}_{n}\left(W_{\alpha} ; \mathbf{x}, \mathbf{y}\right)=\int_{[-1,1]^{d+1}} C_{2 n}^{\left(|\alpha|_{1}+(d+1) / 2\right)} & \left(\sqrt{x_{1} y_{1}} t_{1}+\ldots+\sqrt{x_{d+1} y_{d+1}} t_{d+1}\right) \\
\times & \prod_{i=1}^{d+1} c_{\alpha_{i}}\left(1-t_{i}^{2}\right)^{\alpha_{i}-1} d \mathbf{t} / 2^{d+1} .
\end{aligned}
$$

Proof. The proof follows from Theorem 2.2 upon using (1.3) and the identity

$$
(2 k+\lambda) C_{2 k}^{(\lambda)}(x)=\lambda\left[C_{2 k}^{(\lambda+1)}(x)-C_{2 k-2}^{(\lambda+1)}(x)\right], \quad k \geq 0,
$$

where $C_{-2}^{(\lambda+1)}=0([8$, p. 83, $(4.7 .29)])$.

If one of the $\alpha_{i}=0$ in the above two theorems, then the formula holds upon using the following limit:

$$
\lim _{\mu \rightarrow 0} c_{\mu} \int_{-1}^{1} f(t)\left(1-t^{2}\right)^{\alpha-1} d t=[f(1)+f(-1)] / 2,
$$

where we recall that $c_{\mu}=1 / \int_{-1}^{1}\left(1-t^{2}\right)^{\mu-1} d t$. In particular, when $\alpha=0$, we end up with the following formula:

Corollary 2.4. For $\alpha=0$, the $n$-th reproducing kernel $\mathbf{P}_{n}\left(W_{\alpha} ; \cdot, \cdot\right)$ of $\mathcal{V}_{n}^{d}$ is given by

$$
\begin{aligned}
& \mathbf{P}_{n}\left(W_{0} ; \mathbf{x}, \mathbf{y}\right) \\
& \quad=\frac{2 n+(d-1) / 2}{2^{d}(d-1)} \sum_{\varepsilon \in\{-1,1\}^{d+1}} C_{2 n}^{((d-1) / 2)}\left(\sqrt{x_{1} y_{1}} \varepsilon_{1}+\ldots+\sqrt{x_{d+1} y_{d+1}} \varepsilon_{d+1}\right),
\end{aligned}
$$

where $\mathbf{x}, \mathbf{y} \in \Sigma^{d}, x_{d+1}=1-|\mathbf{x}|_{1}$ and $y_{d+1}=1-|\mathbf{y}|_{1}$.

Since $\mathbf{P}_{n}(\mathbf{x}, \mathbf{x})$ is nonnegative by definition, the formula in Theorem 2.2 leads to a positive integral of a Gegenbauer polynomial; in the case $\alpha=0$, the formula in Corollary 2.4 leads to a nonnegative sum that seems to be of independent interest.

Corollary 2.5. For $\mathrm{x} \in \Sigma^{d}$

$$
\sum_{\varepsilon \in\{-1,1\}^{d+1}} C_{2 n}^{((d-1) / 2)}\left(x_{1} \varepsilon_{1}+\ldots+x_{d} \varepsilon_{d}+\left(1-|\mathbf{x}|_{1}\right) \varepsilon_{d+1}\right) \geq 0 .
$$

For $d=1$, the positivity follows easily from the fact that $C_{2 n}^{(\lambda)}(t)$ attains its maximum at $t=1$ and $t=-1$. For $d>1$, however, the positivity seems to be non-trivial. 


\section{Proof of the theorems}

Recall the definition of $S_{n}\left(W_{\alpha} ; f\right)$ in (1.4). We denote by $S_{n}^{\delta}\left(W_{\alpha} ; f\right)$ the Cesàro $(C, \delta)$ means of $S_{n}(W, f)$, and by $\mathbf{K}_{n}^{\delta}\left(W_{\alpha} ; \cdot, \cdot\right)$ the $(C, \delta)$ means of the orthogonal polynomial series on $\Sigma^{d}$; i.e.,

$\mathbf{K}_{n}^{\delta}\left(W_{\alpha}\right)=\frac{1}{\left(\begin{array}{c}n+\delta \\ n\end{array}\right)} \sum_{k=0}^{n}\left(\begin{array}{c}n-k+\delta \\ n-k\end{array}\right) \mathbf{P}_{k}\left(W_{\alpha}\right)=\frac{1}{\left(\begin{array}{c}n+\delta \\ n\end{array}\right)} \sum_{k=0}^{n}\left(\begin{array}{c}n-k+\delta-1 \\ n-k\end{array}\right) \mathbf{K}_{k}\left(W_{\alpha}\right)$,

so that, by the formula (1.4), we can write

$$
S_{n}^{\delta}\left(W_{\alpha} ; f\right)=\int_{\Sigma^{d}} f(\mathbf{y}) \mathbf{K}_{n}^{\delta}\left(W_{\alpha} ; \mathbf{x}, \mathbf{y}\right) W_{\alpha}(\mathbf{y}) d \mathbf{y} .
$$

For the proof of the theorems, we will try to connect $\mathbf{K}_{n}^{\delta}\left(W_{\alpha}\right)$ with the corresponding kernel for the series of Jacobi polynomials. Let $P_{n}^{(\lambda, \mu)}$ denote the Jacobi polynomials as in [8, Chapt. 4] and let $p_{n}^{(\lambda, \mu)}$ denote the orthonormal Jacobi polynomial with respect to the normalized weight function $w^{(\lambda, \mu)}(t)=w_{\lambda, \mu}(1-t)^{\lambda}(1+t)^{\mu}$ on $[-1,1]$, where $w_{\lambda, \mu}$ is a constant so that $\int_{-1}^{1} w^{(\lambda, \mu)}(t) d t=1$. The polynomial $p_{n}^{(\lambda, \mu)}$ differs from $P_{n}^{(\lambda, \mu)}$ by a constant (cf. [8, p. 68, (4.3.4)]). A function $f \in L^{2}\left(w^{(\lambda, \mu)},[-1,1]\right)$ can be expanded into Jacobi polynomials, whose $n$-partial sum takes the form

$$
s_{n}\left(w^{(\lambda, \mu)} ; f\right)=\int_{-1}^{1} f(y) K_{n}\left(w^{(\lambda, \mu)} ; x, y\right) w^{(\lambda, \mu)}(y) d y,
$$

where the $n$-th reproducing kernel $K_{n}\left(w^{(\lambda, \mu)}\right)$ is given by

$$
K_{n}\left(w^{(\lambda, \mu)} ; x, y\right)=\sum_{k=0}^{n} p_{k}^{(\lambda, \mu)}(x) p_{k}^{(\lambda, \mu)}(y) .
$$

We will also write $K_{n}^{\delta}\left(w^{(\lambda, \mu)}\right)$ for the $(C, \delta)$ means of $K_{n}\left(w^{(\lambda, \mu)}\right)$. The following integration formula plays an essential role in the proof; it helps to connect the summability for $W_{\alpha}$ on $\Sigma^{d}$ to that of Jacobi weights on $[-1,1]$.

Lemma 3.1. Let $g: \mathbb{R} \mapsto \mathbb{R}$ be a polynomial and $V$ be the intertwining operator. Then

$$
\int_{S^{d}} V g(\langle\mathbf{x}, \cdot\rangle)(\mathbf{y}) h_{\alpha}^{2}(\mathbf{y}) d \omega=B_{\alpha} \int_{-1}^{1} g(t|\mathbf{x}|)\left(1-t^{2}\right)^{|\alpha|_{1}+\frac{d-2}{2}} d t,
$$

where $B_{\alpha}=H_{\alpha}^{-1} c_{|\alpha|_{1}+d / 2}$.

This formula is proved in [11] for $h$-harmonics associated with any reflection group, and is used to study the summability of the $h$-harmonic series. It is a nontrivial formula even for $h_{\alpha}$ defined in (1.6); the reader is invited to write the formula out explicitly for the $V f$ defined in (2.5).

Proof of Theorem 1.1. Since $S_{n}^{\delta}\left(W_{\alpha}, f\right)$ is a linear operator, it suffices to prove that

$$
\mathcal{I}_{n}^{\delta}(\mathbf{x}):=\int_{\Sigma^{d}}\left|\mathbf{K}_{n}^{\delta}\left(W_{\alpha} ; \mathbf{x}, \mathbf{y}\right)\right| W_{\alpha}(\mathbf{y}) d \mathbf{y}=\int_{S^{d}}\left|\mathbf{K}_{n}^{\delta}\left(W_{\alpha} ; \mathbf{x},\{\mathbf{y}\}^{2}\right)\right| h_{\alpha}^{2}\left(\mathbf{y}^{\prime}\right) d \omega\left(\mathbf{y}^{\prime}\right)
$$

is uniformly bounded for $\mathbf{x} \in \Sigma^{d}$ if and only if $\delta>|\alpha|_{1}+(d-1) / 2$, where the second equal sign follows from the formula (2.2), and we use the notation $\mathbf{y}^{\prime}=\left(\mathbf{y}, y_{d+1}\right) \in$ 
$S^{d}$ as in (2.3). First we relate the kernel $\mathbf{K}_{n, d}^{\delta}\left(W_{\alpha}\right)$ to that of Jacobi polynomials. From $[8,(4.3 .4)$ and (4.1.5)] it is easy to verify that

$$
\frac{2 n+\lambda}{\lambda} C_{2 n}^{(\lambda)}(t)=p_{n}^{(\lambda-1 / 2,-1 / 2)}(1) p_{n}^{(\lambda-1 / 2,-1 / 2)}\left(2 t^{2}-1\right) .
$$

Using the above formula, it follows from (2.7), (1.3) and (1.5) that we can write

$$
\mathbf{K}_{n}^{\delta}\left(W_{\alpha} ; \mathbf{x},\{\mathbf{y}\}^{2}\right)=2^{-d-1} \sum_{\varepsilon \in G} V\left[K_{n}^{\delta}\left(w^{\left(|\alpha|_{1}+(d-2) / 2,-1 / 2\right)} ; 1,2\langle\cdot, \mathbf{z}(\varepsilon)\rangle^{2}-1\right)\right]\left(\mathbf{y}^{\prime}\right),
$$

where $\mathbf{z}(\varepsilon)=\left\{\mathbf{x}^{\prime}\right\}^{1 / 2} \varepsilon$. We observe that $|\mathbf{z}(\varepsilon)|^{2}=\left|\mathbf{x}^{\prime}\right|_{1}=1$. Hence, since $V$ defined in (2.5) is clearly a positive operator, we can use (3.4) of Lemma 3.1 to conclude that

$$
\begin{aligned}
\mathcal{I}_{n}^{\delta}(\mathbf{x}) & \leq 2^{-d-1} \sum_{\varepsilon \in G} \int_{S^{d}} V\left[\left|K_{n}^{\delta}\left(w^{\left(|\alpha|_{1}+(d-2) / 2,-1 / 2\right)} ; 1,2\langle\cdot, \mathbf{z}(\varepsilon)\rangle^{2}-1\right)\right|\right]\left(\mathbf{y}^{\prime}\right) h_{\alpha}^{2}\left(\mathbf{y}^{\prime}\right) d \omega \\
& =B_{\alpha} \int_{-1}^{1}\left|K_{n}^{\delta}\left(w^{\left(|\alpha|_{1}+(d-2) / 2,-1 / 2\right)} ; 1,2 t^{2}-1\right)\right|\left(1-t^{2}\right)^{|\alpha|_{1}+\frac{d-2}{2}} d t \\
& =\int_{-1}^{1}\left|K_{n}^{\delta}\left(w^{\left(|\alpha|_{1}+(d-2) / 2,-1 / 2\right)} ; 1, u\right)\right| w^{\left(|\alpha|_{1}+\frac{d-2}{2},-1 / 2\right)}(u) d u,
\end{aligned}
$$

where the last step follows from a change of variable, and the constant in front of the integral becomes 1 as can be determined by setting $n=0$. The last integral is bounded if and only if $\delta>|\alpha|_{1}+(d-1) / 2$, since it is precisely the condition on the $(C, \delta)$ summability of the Jacobi series for $w^{\left(|\alpha|_{1}+(d-2) / 2,-1 / 2\right)}$ at the point $x=1$, and the conclusion follows from [8, Theorem 9.1.3, p. 246].

In order to prove that the condition on the index is also necessary, we need more detailed information on $\mathbf{K}_{n}^{\delta}\left(W_{\alpha}\right)$. Let us first assume that $|\alpha|_{1} \neq 0$ and assume, without loss of generality, that $\alpha_{1}>0$. We prove that $\mathcal{I}_{n}^{\delta}\left(\mathbf{e}_{1}\right)$ is unbounded for $\delta=|\alpha|_{1}+(d-1) / 2$, where $\mathbf{e}_{1}=(1,0, . ., 0)$. From Theorem 2.2 and $(3.5)$, we have

$$
\begin{aligned}
\mathbf{P}_{n}\left(W_{\alpha} ; \mathbf{e}_{1}, \mathbf{y}\right)= & c_{\alpha_{1}} p_{n}^{\left(|\alpha|_{1}+(d-2) / 2,-1 / 2\right)}(1) \\
& \times \int_{-1}^{1} p_{n}^{\left(|\alpha|_{1}+(d-2) / 2,-1 / 2\right)}\left(2 y_{1} t^{2}-1\right)(1-t)^{\alpha_{1}-1} d t .
\end{aligned}
$$

Using (1.3), (1.5) and (3.3), we conclude that

$$
\mathbf{K}_{n}^{\delta}\left(W_{\alpha} ; \mathbf{e}_{1}, \mathbf{y}\right)=c_{\alpha_{1}} \int_{-1}^{1} K_{n}^{\delta}\left(w^{\left(|\alpha|_{1}+(d-2) / 2,-1 / 2\right)} ; 1,2 y_{1} t^{2}-1\right)(1-t)^{\alpha_{1}-1} d t
$$

Since the right hand side depends only on $y_{1}$, its integral against $W_{\alpha}(\mathbf{y}) d \mathbf{y}$ over $\Sigma^{d}$ can be reduced to an integral over $[0,1]$ by changing variables $y_{2}=\left(1-x_{1}\right) u_{2}, \ldots$, $y_{d}=\left(1-x_{1}\right) u_{d}$, where $1-|\mathbf{y}|_{1}=\left(1-y_{1}\right)\left(1-u_{2}-\ldots-u_{d}\right)$. This way, we conclude that

$$
\begin{aligned}
\mathcal{I}_{n}^{\delta}\left(\mathbf{e}_{1}\right)=\operatorname{const} \int_{0}^{1} & \int_{-1}^{1}\left|K_{n}^{\delta}\left(w^{\left(|\alpha|_{1}+(d-2) / 2,-1 / 2\right)} ; 1,2 y_{1} t^{2}-1\right)\right| \\
& \times(1-t)^{\alpha_{1}-1} d t y_{1}^{\alpha_{1}-1 / 2}\left(1-y_{1}\right)^{\left|\alpha_{1}\right|-\alpha_{1}+(d-2) / 2} d y_{1},
\end{aligned}
$$

where const is a constant whose value can be determined by setting $n=0$. We change the inner integral from $[-1,1]$ to $[0,1]$ so that we can make the change of 
variable $t^{2} \mapsto u / y_{1}$; then interchange the order of integrals to get that

$$
\begin{gathered}
\mathcal{I}_{n}^{\delta}\left(\mathbf{e}_{1}\right)=\mathrm{const} \int_{0}^{1}\left|K_{n}^{\delta}\left(w^{\left(|\alpha|_{1}+(d-2) / 2,-1 / 2\right)} ; 1,2 u-1\right)\right| \int_{u}^{1}\left(y_{1}-u\right)^{\alpha_{1}-1} \\
\times\left(1-y_{1}\right)^{\left|\alpha_{1}\right|-\alpha_{1}+(d-2) / 2} d y_{1} u^{-1 / 2} d u .
\end{gathered}
$$

The inner integral is a beta integral which is equal to a constant multiple of the function $(1-u)^{|\alpha|_{1}+(d-2) / 2}$ upon the change of variable $s=\left(y_{1}-u\right) /(1-u)$; finally letting $u \mapsto 2 v-1$, we conclude that

$$
\mathcal{I}_{n}^{\delta}\left(\mathbf{e}_{1}\right)=\int_{-1}^{1}\left|K_{n}^{\delta}\left(w^{\left(|\alpha|_{1}+(d-2) / 2,-1 / 2\right)} ; 1, v\right)\right| w^{\left(|\alpha|_{1}+(d-2) / 2,-1 / 2\right)}(v) d v,
$$

where the constant in front of the integral is 1 , which is determined by setting $n=0$. This integral also holds for $\alpha=0$ upon taking the limit $|\alpha|_{1} \rightarrow 0$. That the integral is unbounded for $\delta=|\alpha|_{1}+(d-1) / 2$ again follows from [8, p. 246].

Proof of Theorem 1.2. Using the formula (3.6) and the fact that $V$ is a positive operator, we need to show that $K_{n}^{\delta}\left(w^{\left(|\alpha|_{1}+(d-2) / 2,-1 / 2\right)} ; 1, t\right) \geq 0$ for $\delta=|\alpha|_{1}+$ $(d+1) / 2$ and $-1 \leq t \leq 1$ in order to prove the first part of the statement. That is, we need to prove that the $(C, \lambda+1)$ means

$$
\begin{aligned}
K_{n}^{\lambda+1} & \left(w^{(\lambda-1 / 2,-1 / 2)} ; 1, t\right) \\
\quad= & \sum_{k=0}^{n}\left(\begin{array}{c}
n-k+\lambda+1 \\
n-k
\end{array}\right) p_{k}^{(\lambda-1 / 2,-1 / 2)}(1) p_{k}^{(\lambda-1 / 2,-1 / 2)}(t) \geq 0
\end{aligned}
$$

with $\lambda=|\alpha|_{1}+(d-1) / 2$. This inequality, however, is a special case of the AskeyGasper inequality for Jacobi polynomials $P_{n}^{(\alpha, \beta)}$, proved by Gasper in $[6$, Theorem 3 , p. 434] (see also [1]), which states that the $(C, \alpha+\beta+2)$ means of the Jacobi series is nonnegative.

To prove the second part of the statement, we notice that it follows from (3.7) that

$$
\sigma_{n}^{\delta}:=\mathbf{K}_{n}^{\delta}\left(W_{\alpha} ; \mathbf{e}_{1}, 0\right)=K_{n}^{\delta}\left(w^{(\lambda-1 / 2,-1 / 2)} ; 1,-1\right), \quad \lambda=|\alpha|_{1}+(d-1) / 2 .
$$

Since the positivity of the means implies uniform convergence, it follows from Theorem 1.1 that a necessary condition for the positivity is $\delta>\lambda$. Let us consider the $(C, \lambda)$ means first. From (1.5), (3.3) and the formulae (4.1.1) and (4.3.4) in [8], we have

$$
\begin{aligned}
& \left(\begin{array}{c}
n+\lambda \\
n
\end{array}\right) \sigma_{n}^{\lambda}=\sum_{k=0}^{n}\left(\begin{array}{c}
n-k+\lambda \\
\lambda
\end{array}\right) p_{k}^{(\lambda-1 / 2,-1 / 2)}(1) p_{k}^{(\lambda-1 / 2,-1 / 2)}(-1) \\
& \quad=\sum_{k=0}^{n}(-1)^{k}\left(\begin{array}{c}
n-k+\lambda \\
\lambda
\end{array}\right) \frac{(2 k+\lambda) \Gamma(k+\lambda)}{\Gamma(k+1) \Gamma(\lambda+1)} \\
& \quad=\sum_{k=0}^{n}(-1)^{k}\left(\begin{array}{c}
n-k+\lambda \\
\lambda
\end{array}\right)\left(\begin{array}{c}
k+\lambda \\
\lambda
\end{array}\right)+\sum_{k=0}^{n-1}(-1)^{k}\left(\begin{array}{c}
n-1-k+\lambda \\
\lambda
\end{array}\right)\left(\begin{array}{c}
k+\lambda \\
\lambda
\end{array}\right) \\
& \quad=\frac{1+(-1)^{n}}{2}\left(\begin{array}{c}
\lambda+n / 2 \\
\lambda
\end{array}\right)-\frac{1+(-1)^{n-1}}{2}\left(\begin{array}{c}
\lambda+\left(\begin{array}{c}
n-1) / 2 \\
\lambda
\end{array}\right),
\end{array}\right.
\end{aligned}
$$

where the last equation follows from [7, Sec. 4.2.5., \#38]. In particular, this shows that $\sigma_{2 j}^{\lambda}=-\sigma_{2 j+1}^{\lambda}=\left(\begin{array}{c}\lambda+j \\ \lambda\end{array}\right)$, so that $\sigma_{2 j+1}^{\lambda}$ is negative. Moreover, if $n$ is odd, say, 
$n=2 m+1$, then for $0<\mu<1$ we have $([13,(1.10)$, p.77]),

$$
\begin{aligned}
& \sigma_{n}^{\lambda+\mu}=\sum_{k=0}^{n}\left(\begin{array}{c}
n-k+\mu-1 \\
\mu-1
\end{array}\right) \sigma_{k}^{\lambda} \\
& =\sum_{j=0}^{m}\left[\left(\begin{array}{c}
2 m-2 j+\mu-1 \\
\mu-1
\end{array}\right)-\left(\begin{array}{c}
2 m-2 j+\mu-2 \\
\mu-1
\end{array}\right)\right]\left(\begin{array}{c}
\lambda+j \\
j
\end{array}\right) \\
& =(\mu-1) \sum_{j=0}^{m} \frac{\Gamma(2 m-2 j+\mu-1)}{\Gamma(\mu) \Gamma(2 m-2 j+1)}\left(\begin{array}{c}
\lambda+j \\
j
\end{array}\right)<0 .
\end{aligned}
$$

This completes the proof.

\section{REFERENCES}

1. R. Askey, Orthogonal polynomials and special functions, SIAM, Philadelphia, 1975. MR 58:1288

2. H. Berens and Y. Xu, Fejér means for multivariate Fourier series, Math. Z. 221 (1996), 449-465. MR 97a:42003

3. C. Dunkl, Orthogonal polynomials with symmetry of order three, Can. J. Math. 36 (1984), 685-717. MR 86h:33003

4. C. Dunkl, Differential-difference operators associated to reflection groups, Trans. Amer. Math. Soc. 311 (1989), 167-183; errata, Math. Comp. 66 (1997), 1765-1766. MR 90k:33027

5. C. Dunkl, Integral kernels with reflection group invariance, Can. J. Math. 43 (1991), 12131227. MR 93g:33012

6. G. Gasper, Positive sums of the classical orthogonal polynomials, SIAM J. Math. Anal. 8 (1977), 423-447. MR 55:5925

7. A. P. Prudnikov, Yu. A. Brychkov and O. I. Marichev, Integrals and Series, Vol. 1: Elementary Functions, Gordon and Breach Sci. Publ., New York, 1986. MR 88f:00013; CMP 97:08

8. G. Szegö, Orthogonal polynomials, 4th ed., Amer. Math. Soc. Colloq. Publ. vol.23, Providence, RI, 1975. MR 51:8724

9. Y. Xu, On orthogonal polynomials in several variables, Special functions, $q$-series, and related topics, Fields Institute Communications Series, vol. 14, 1997, pp. 247-270. CMP 97:12

10. Y. Xu, Orthogonal polynomials for a family of product weight functions on the spheres, Canadian J. Math. 49 (1997), 175-192. CMP 97:09

11. Y. Xu, Integration of the intertwining operator for h-harmonic polynomials associated to reflection groups, Proc. Amer. Math. Soc. 125 (1997), 2963-2973. MR 97m:33004

12. Y. Xu, Orthogonal polynomials and cubature formulae on spheres and on simplices (to appear).

13. A. Zygmund, Trigonometric Series, 2nd ed., Cambridge Univ. Press, Cambridge, 1968. MR 38: 4882

Department of Mathematics, University of Oregon, Eugene, Oregon 97403-1222

E-mail address: yuan@math.uoregon.edu 